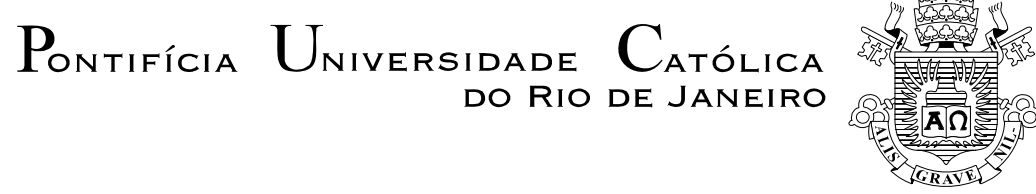

Luiz de Magalhães Ozorio

Opções Reais na Siderurgia: O caso Brasileiro

\author{
Tese de Doutorado
}

Tese apresentada ao Programa de Pós-graduação em Engenharia de Produção do Departamento de Engenharia Industrial da PUC-Rio como parte dos requisitos parciais para obtenção do título de Doutor em Engenharia de Produção

Orientador: Prof. Tara Keshar Nanda Baidya

Rio de Janeiro

Agosto de 2010 


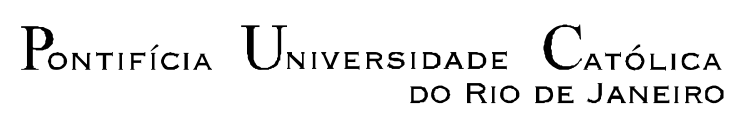

Luiz de Magalhães Ozorio

\section{Opções Reais na Siderurgia: 0 caso Brasileiro}

Tese apresentada como requisito parcial para obtenção do grau de Doutor pelo Programa de Pós-Graduação em Engenharia de Produção da PUC-Rio. Aprovada pela Comissão Examinadora abaixo assinada.

Prof. Tara Keshar Nanda Baidya

Orientador

Departamento de Engenharia Industrial - PUC-Rio

Prof. Marco Antonio Guimarães Dias

Departamento de Engenharia Industrial - PUC-Rio

Prof. Carlos de Lamare Bastian Pinto IBMEC

Prof. Paulo Henrique Soto Costa

Departamento de Engenharia Industrial - PUC-Rio

Prof. Marcelo de Sales Pessoa

IPEA

Prof. Fernando Antonio Lucena Aiube Departamento de Engenharia Industrial - PUC-Rio

Prof. Adrian Heringer Pizzinga

UFF

Prof. Luiz Eduardo Teixeira Brandão

Departamento de Administração - PUC-Rio

Prof. José Eugenio Leal Coordenador Setorial do Centro Técnico Científico - PUC-Rio

Rio de Janeiro, 19 de agosto de 2010 
Todos os direitos reservados. É proibida a reprodução total ou parcial do trabalho sem autorização da universidade, do autor e do orientador.

\section{Luiz de Magalhães Ozorio}

Graduado em Administração de Empresas pela FIB (1996), MBA em Finanças pelo Ibmec/RJ (1999) e Mestre em Engenharia de Produção com foco na Análise de Projetos Industriais e Tecnológicos pela COPPE/UFRJ (2002). Professor da Faculdade de Economia e dos Programas de Pós-Graduação do Ibmec/RJ. Consultor na Área de Finanças Corporativas tendo atuado em projetos em renomadas instituições e empresas, como: Banco Mundial, Petrobras, Eletrobrás, Vale, Bradesco Seguros, Ampla, Light, Banco do Nordeste, Petros, Postalis, Agora Corretora, Souza Cruz, Amil, entre outras.

Ficha Catalográfica

Ozorio, Luiz de Magalhâes

Opções reais na siderurgia: o caso brasileiro / Luiz de Magalhâes Ozorio ; orientador: Tara Keshar Nanda Baidya. $-2010$.

$208 \mathrm{f.} \mathrm{;} 30 \mathrm{~cm}$

Tese (doutorado) - Pontifícia Universidade Católica do Rio de Janeiro, Departamento de Engenharia Industrial, 2010.

Inclui bibliografia

1. Engenharia Industrial - Teses. 2. Opções reais. 3. Processos estocásticos. 4. Reversão à média. 5. Método binomial. 6. Simulação de Monte Carlo. 7. Preço do aço. 8. Indústria siderúrgica. I. Baidya, Tara Keshar Nanda. II. Pontifícia Universidade Católica do Rio de Janeiro. Departamento de Engenharia Industrial. III. Título. 
Dedico esta tese a minha esposa Julia e aos meus pais Luiz e Lêda, meus maiores incentivadores. 


\section{Agradecimentos}

Ao meu orientador, professor Tara Keshar Nanda Baidya, pela confiança, oportunidades, atenção e sua permanente preocupação com o meu desenvolvimento.

Aos professores Luiz Brandão e Marco Antonio Dias, por suas aulas, participações nos processos de qualificação e tese de doutorado e todas as importantes contribuições ao meu trabalho.

Aos professores Carlos Bastian e Adrian Pizzinga pela imensurável contribuição ao meu desenvolvimento, disponibilidade de tempo e importantes contribuições e efetuadas ao meu trabalho.

Aos professores Paulo Henrique Soto, Fernando Aiube e Marcelo Pessoa, pela revisão do meu trabalho, pelos prestimosos comentários e contribuições na minha defesa de tese.

À minha colega de doutorado e amiga Frances Blank, pelo convívio nestes anos de estudo e todo apoio nos estudos para qualificação e desenvolvimento da tese.

Às funcionárias do DEI - Fernanda, Isabel, Ana e em especial à Claudinha - pelo profissionalismo, solidariedade e por estarem sempre dispostas em me ajudar nos assuntos administrativos.

À minha querida família, minha esposa Júlia, minha mãe Lêda, meus sogros Alvaro e Telma, meus irmãos Diego e Priscila, Lila e Luiz, e Pedro, e meus sobrinhos Lucas, Lígia e Luiza, pelo amor, incentivo e compreensão ao longo de todos esses anos.

Ao meu maior professor e amigo, meu pai Luiz, quem sempre me incentivou em todos os mais importantes projetos de minha vida. 


\section{Resumo}

Ozorio, Luiz de Magalhães; Baidya, Tara Keshar Nanda (Orientador). Opções Reais na Siderurgia: O Caso Brasileiro. Rio de Janeiro, 2010. 208p. Tese de Doutorado - Departamento de Engenharia Industrial, Pontifícia Universidade Católica do Rio de Janeiro.

O presente trabalho analisa a aplicabilidade do método das opções reais na avaliação de projetos de investimento no setor siderúrgico e compara os resultados obtidos com os métodos tradicionais de avaliação de projetos. Por ser o aço uma commodity de grande oscilação nos preços e variabilidade nas quantidades comercializadas, os retornos dos projetos siderúrgicos são voláteis. A presença das incertezas citadas, combinadas ao conjunto de flexibilidades gerenciais existentes nos processos produtivos de aço, gera ambiente propício ao surgimento de valiosas opções reais, que - por ser assim - podem alterar significativamente o valor dos projetos e a tomada de decisão de investimentos na siderurgia. Além de contemplar o reconhecimento das principais opções reais existentes na siderurgia e prescrever métodos para avaliá-las, o trabalho analisa os modelos estocásticos que demonstram a melhor adequação ao comportamento dos preços do aço, ao levar em consideração a disponibilidade de dados empíricos existentes no mercado. Como aplicações das metodologias discutidas no decorrer da tese são apresentadas avaliações de dois tipos distintos de opções em reais em projetos siderúrgicos - parada temporária em fornos elétricos e troca de produto em siderúrgicas integradas - utilizando 3 tipos distintos de processos estocásticos: MGB, MRM e MRM com Tendência. Os resultados obtidos demonstraram que as flexibilidades gerenciais avaliadas podem agregar significativamente o valor criado em projetos siderúrgicos, sendo possível também observar a importância da escolha do processo estocástico tanto no valor do projeto como também no valor da opção.

\section{Palavras-chave}

Opções Reais, Processos Estocásticos, Reversão à Média, Método Binomial, Simulação de Monte Carlo, Preço do Aço, Indústria Siderúrgica. 


\section{Abstract}

Ozorio, Luiz de Magalhães; Baidya, Tara Keshar Nanda (Advisor). Real Options Analysis of Steel Sector Projects: The Brazilian Case. Rio de Janeiro, 2010. 208p. DSc. Thesis - Departamento de Engenharia Industrial, Pontifícia Universidade Católica do Rio de Janeiro.

This work analyzes the applicability of the real options method in the valuation of investments in steel industry, and compares the outcomes with the traditional methods of project evaluation. Being steel a commodity with high price volatility and great changeability in its demand, the return of steel projects are stochastic. The presence of these uncertainties, combined with the existing set of managerial flexibilities in the steel productive processes, create an adequate environment to emerge valuable real options that consequently may interfere significantly with the project values and the decision making process in the steel industry investments. Besides contemplating the recognition of the main existing real options in the steel sector and prescribing methodologies to evaluate them, the work analyzes stochastic methods that show the best fit to steel price performance, considering the available empirical data existing in the market. As application of the discussed methodologies in the course of the thesis the evaluation of two diferent kinds of real options in the steel industry are presented - shut down options in electrical furnace plants and switch of product in integrated plants using three different types of sthocastic processes: GBM, MRM and MRM with Drift. The obtained results showed that the evaluated managerial flexibilities can agregate significantily the created value in steel projects and it also may be observed the relevance of the choice of stochastic process not only in the projects value but in the options value as well.

\section{Keywords}

Real Options, Stochastic Processes, Mean Reversion, Binomial Lattice, Monte Carlo Simulation, Steel Prices, Steel Sector. 


\section{Sumário}

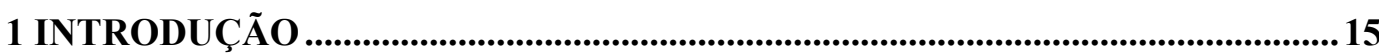

2 VISÃO GERAL DO SETOR SIDERÚRGICO ....................................................19

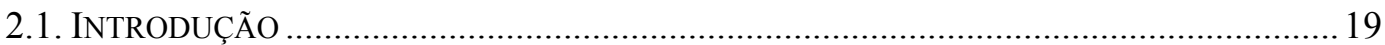

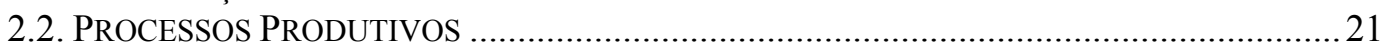

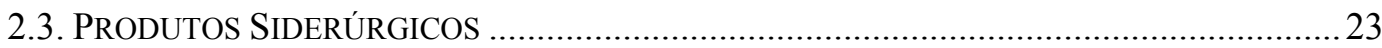

2.4. A SIDERURGIA NO MUNDO E O SETOR SIDERÚRGICO BRASILEIRO..............................26

2.5. PERFIL DAS PRINCIPAIS EMPRESAS SIDERÚRGICAS BRASILEIRAS ................................35

2.6. RISCOS INERENTES AO SETOR SIDERÚRGICO E AS FLEXIBILIDADES

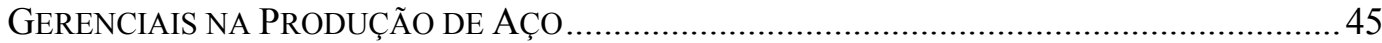

\section{PROCESSOS ESTOCÁSTICOS E APLICAÇÕES EM PROJETOS NA}

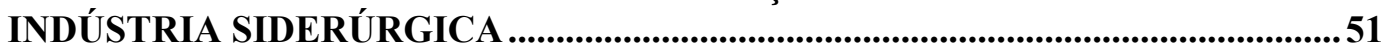

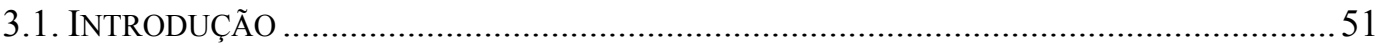

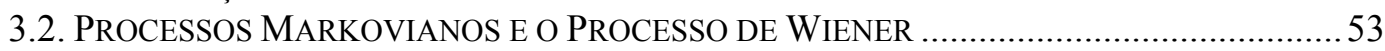

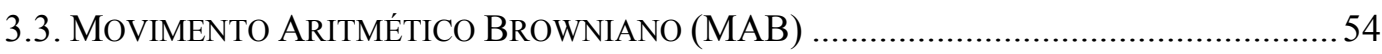

3.4. PROCESSOS DE ITÔ E MOVIMENTO GEOMÉTRICO BROWNIANO (MGB) ......................55

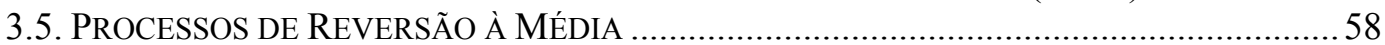

3.5.1. Movimento de Reversão à Média Aritmético (MRA) de Ornstein-Uhlenbeck........58

3.5.2. Modelo de Reversão à Média Geométrico (MRG) de Dixit \& Pindyck (1994)...... 61

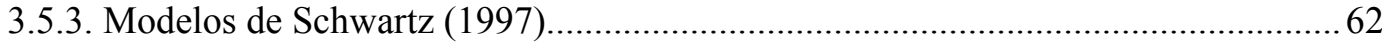

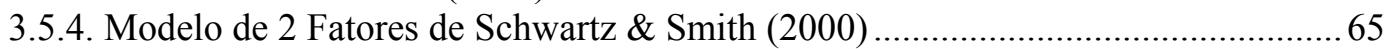

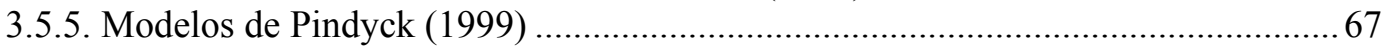

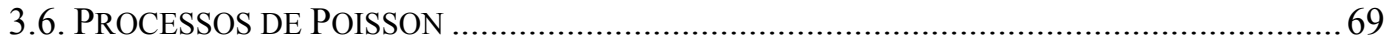

3.7. PROCESSOS MISTOS DE DIFUSÃO COM SALTOS ..................................................... 71

3.8. DisCUSSÃO SOBRE PROCESSOS ESTOCÁSTICOS E A ANÁLISE DE OPÇÕES REAIS

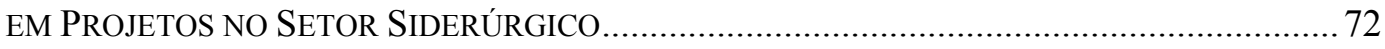

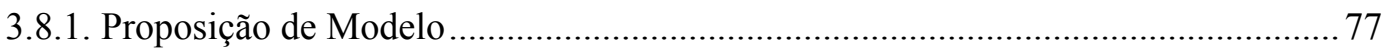

3.8.2. Modelo Empírico: Discretização, Estimação de Parâmetros e Simulação Neutra

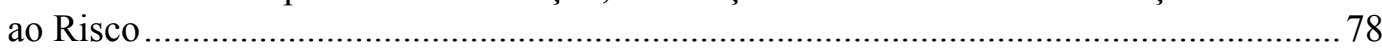

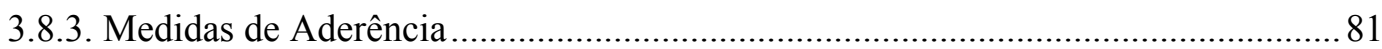

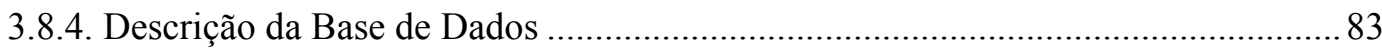

3.8.5. Resultados Empíricos: Parâmetros Estimados, Testes do Processo e Análise

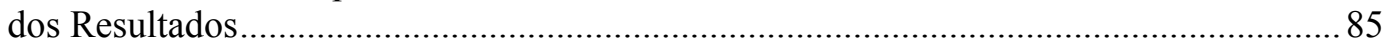

4 METODOLOGIAS DE AVALIAÇÃO DE INVESTIMENTO ................................90

4.1. INTRODUÇÃO: MÉTODOS TRADICIONAIS DE ANÁLISE DE PROJETOS E OS

4.2. COMPARAÇÃo ENTRE O MÉTOdO DO FluXo DE CAIXA DESCONTAdO E A

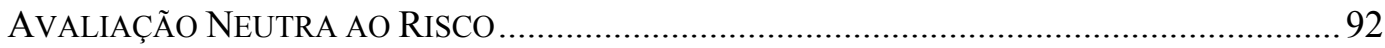

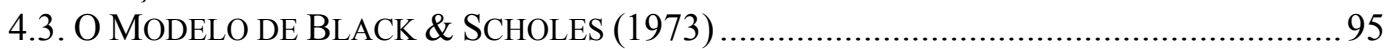

4.4. O MÉTODO BINOMIAL DE COX, RosS \& RUBINSTEIN (1979) ….................................... 96

4.5. MÉTODO BINOMIAL PARA MOVIMENTO DE REVERSÃO À MÉDIA …........................... 98

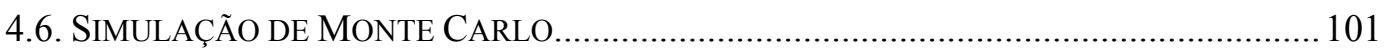

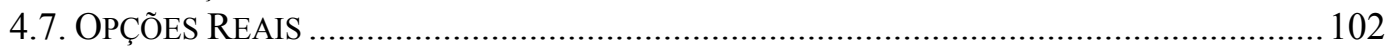

4.7.1. Semelhanças e Diferenças entre Opções Financeiras e Reais ................................ 102

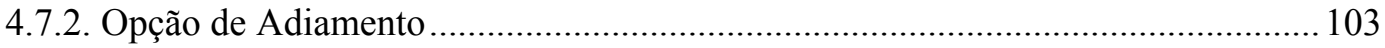

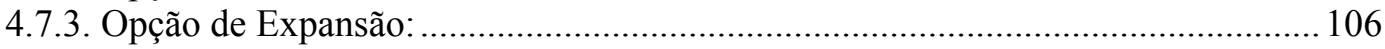

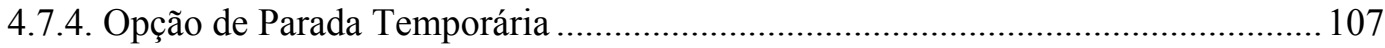

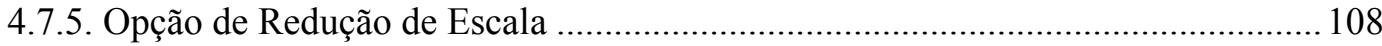




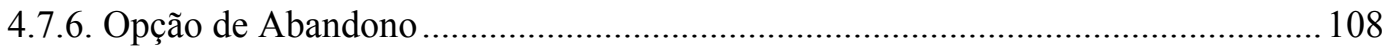

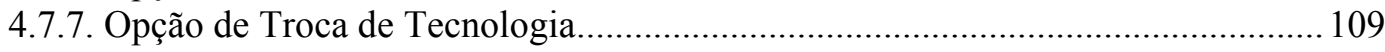

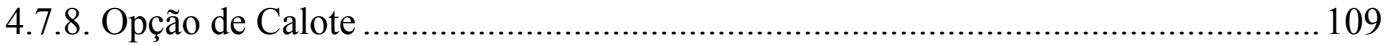

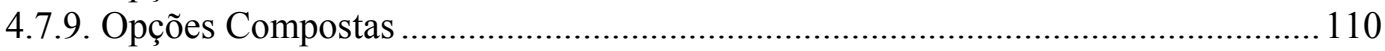

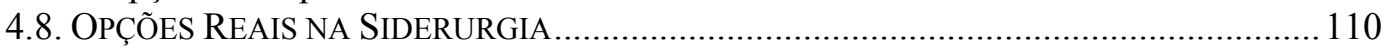

4.8.1. Casos Base 1 - Projeto de Investimento em um Forno-Elétrico (Usina Semi-

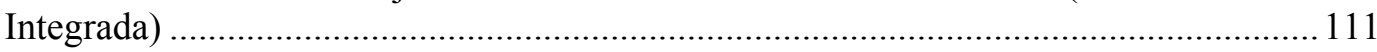

4.8.2. Caso Base 2 - Projeto de Investimento em um Alto-forno (Usina Integrada) ....... 113

4.8.3. Opção de Parada Temporária em Usinas Semi-Integradas ...................................... 114

4.8.3.1. Opção de Parada Temporária em Usinas Siderúrgicas - MGB …………............ 115

4.8.3.2. Opção de Parada Temporária em Usinas Siderúrgicas - MRM ........................... 117

4.8.3.3. Opção de Parada Temporária em Usinas Siderúrgicas - MRM com

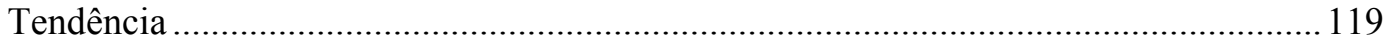

4.8.4. Opção de Troca de Tecnologia em Usinas Integradas .......................................... 120

4.8.4.1. Opção de Troca de Tecnologia em Usinas Integradas - MGB ........................... 121

4.8.4.2. Opção de Troca de Tecnologia em Usinas Integradas - MRM............................ 124

4.8.4.3. Opção de Troca de Tecnologia em Usinas Integradas - MRM com

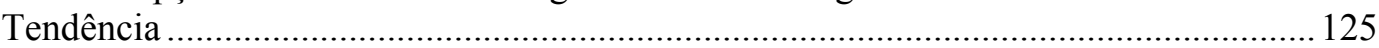

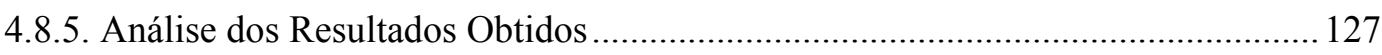

4.8.5.1. Análise da Opção de Parada Temporária em Usinas Siderúrgicas...................... 127

4.8.5.2. Análise da Opção de Troca de Tecnologia em Usinas Integradas ..................... 135

5 CONCLUSÕES E RECOMENDAÇÕES PARA PESQUISAS FUTURAS ..........140

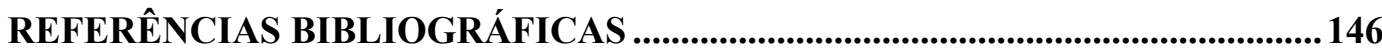

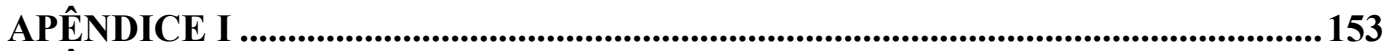

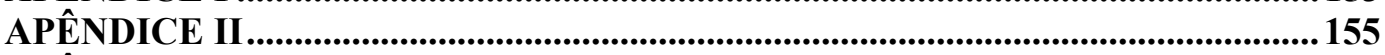

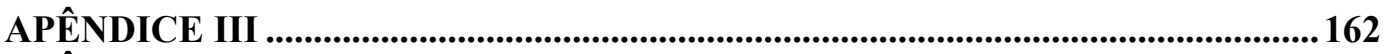

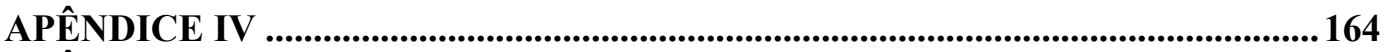

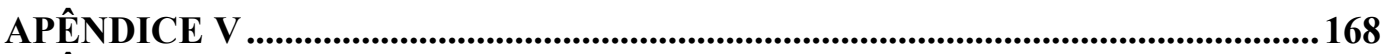

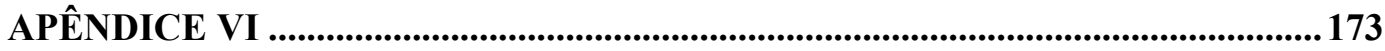

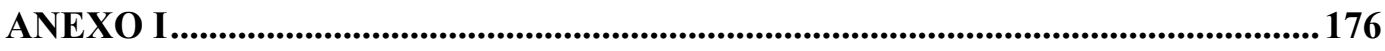

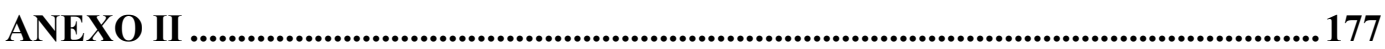

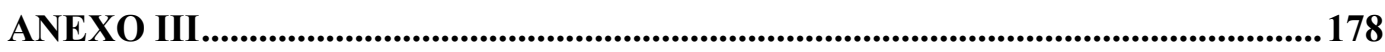

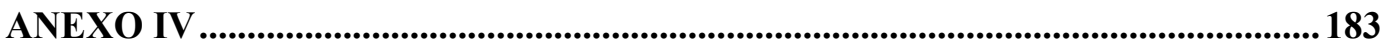

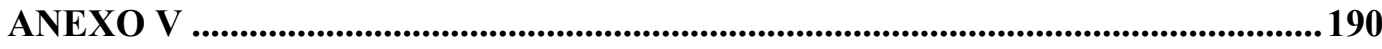

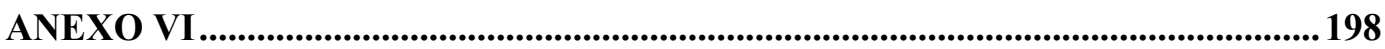

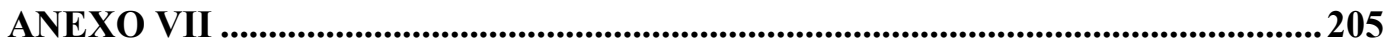




\section{Lista de Figuras}

Figura 1 - Etapas dos Processos Produtivos do Aço - Fluxo Simplificado de Produção.

Figura 2 - Realizações de um Processo Estocástico. 


\section{Lista de Gráficos}

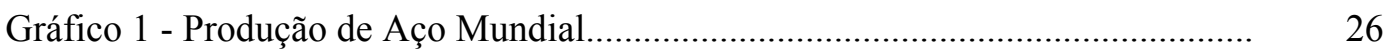

Gráfico 2 - Participação no Consumo Mundial de Aço (\%)....................................... 27

Gráfico 3 - Participação no Setor Brasileiro por Empresa (2009)............................ 32

Gráfico 4 - Segmentação da Demanda por Aço no Brasil - 2009............................. 33

Gráfico 5 - Vendas nos Mercados Interno e Externo da CSN $-1^{\circ}, 2^{\circ}$ e $3^{\circ}$ Trimestres de 2009

Gráfico 6 - Percentual das Vendas por Tipo de Produto da CSN $-2^{\circ}$ e $3^{\circ}$ Trimestre de 2009-11-22.

Gráfico 7 - Produção de Aços Bruto e Laminados da Gerdau - 3T08 até 3T09......

Gráfico 8 - Volume de Vendas por Área de Negócio da Arcelor-Mital Brasil em 1.000 Ton/Ano - 2007 e 2008

Gráfico 9 - Volume de Vendas de Aços Longos da Arcelor-Mital Brasil em 1.000 Ton/Ano - 2007 e 2008

Gráfico 10 - Volume de Vendas de Aços Planios da Arcelor-Mital Brasil em 1.000 Ton/Ano - 2007 e 2008.

Gráfico 11 - Preço Spot (US\$/Ton) da Bobina de Laminado à Quente no Mercado Norte-Americano - jan/2000 até set/2009...

Gráfico 12 - $\Delta$ PIB Mundial X $\Delta$ Producao Mundial de Aço..................................... 47

Gráfico 13 - Placas de aço - Índice de Preços de Placas de Aço................................ 73

Gráfico 14 - LME Steel Billet - Far Fast - 3 months seller..................... 76

Gráfico 15 - Índices de Preços do Aço no Período de Jan/2000 a Abr/2009........... 82

Gráfico 16 - Índices de Preços do Aço deflacionados pelo IGP-DI no Período de $\mathrm{Jan} / 2000$ a Abr/2009.

Gráfico 17 - Índice de Preços Placas de Aço no Período de Jan/1996 a Dez/2009..

Gráfico 18 - Índice de Preços Placas de Aço deflacionado pelo IGP-DI no Período de Jan/1996 a Dez/2009.

Gráfico 19 - Log dos Preços da Série de Placas de Aço (IBS) deflacionada pelo IGP-DI no Período de Jan/2000 a Abr/2009. 


\section{Lista de Tabelas}

Tabela 1 - Produção Mundial de Aço Bruto dos Países associados ao IISI............. 27

Tabela 2 - Maiores Produtores de Aço Bruto do Mundo (Anual) - 106 t................. 28

T abela 3 - Produção de Aço Bruto na América Latina (Anual) - 103 t. 29

Tabela 4 - Ranking dos Países Maiores Exportadores e Importadores de Aço Milhões de Toneladas - 2009 .

Tabela 5 - Brasil - Produção de Aço Bruto (Anual)

Tabela 6 - Brasil - Distribuição Regional da Produção de Aço Bruto em Milhares de Toneladas (Anual).

Tabela 7 - Vendas Mercado Interno......................................................................

Tabela 8 - Vendas Mercado Externo (USINAS) ................................................... 34

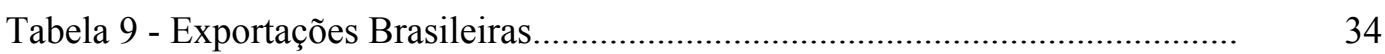

Tabela 10 - Produção Anual das Usinas de Ipatinga e Cubatão - 2003 a 2008....... 40

Tabela 11 - Indicadores operacionais - produção de vendas................................... 40

Tabela 12 - Produção de Aços Bruto e Laminados da Gerdau $-1^{\circ}$, $2^{\circ}$ e $3^{\circ}$ Trimestres de 2009.

Tabela 13 - Parâmetros dos Modelos MGB e MRM estimados a partir das séries de Índices de Preços do Aço (IBS) deflacionados pelo IGP-DI no Período de $\mathrm{Jan} / 2000$ a Abr/2009.

Tabela 14 - Parâmetros do Modelo MRM com Tendência estimados a partir das séries de Índices de Preços do Aço (IBS) deflacionados pelo IGP-DI no Período de Jan/2000 a Abr/2009.

Tabela 15 - Resultados dos Testes de Aderência - Pseudo R2, EQM e MAPE dos 3 Tipos de Processo - MGB, MRM e MRM com Tendência - sobre as 6 Série de Preços de Aço (IBS) deflacionadas pelo IGP-DI no Período de Jan/2000 a Abr/2009.

Tabela 16 - Comparação entre os Parâmetros dos Modelos MGB e MRM estimados a partir das séries do Índice de Preços de Placa de Aços (Bloomberg) no Período de Jan/1996 a Dez/2009 e do Índice de Preços de Placas de Aço (IBS) no Período de Jan/2000 a Abr/2009, ambos deflacionados pelo IGP-DI.......

Tabela 17 - Comparação entre os Parâmetros do Modelo MRM com Tendência estimados a partir das séries do Índice de Preços de Placa de Aços (Bloomberg) no Período de Jan/1996 a Dez/2009 e do Índice de Preços de Placas de Aço (IBS) no Período de Jan/2000 a Abr/2009, ambos deflacionados pelo IGP-DI....... 
Tabela 18 - Comparação dos Resultados dos Testes de Aderência - Pseudo R2, EQM e MAPE - dos 3 Tipos de Processo - MGB, MRM e MRM com Tendência - sobre séries do Índice de Preços de Placa de Aços (Bloomberg) no Período de Jan/1996 a Dez/2009 e do Índice de Preços de Placas de Aço (IBS) no Período de Jan/2000 a Abr/2009, ambos deflacionados pelo IGP-DI.

Tabela 19 - Outros Custos e Despesas (OCD) do projeto nos casos de 90\% de uso da capacidade, parada temporária 1 e 2 .

Tabela 20 - Opção de Parada Temporária: Valor do Projeto, VPL Estático, VPL Expandido e o Valor da Opção em Milhões de Reais para Parada Temporária Tipos 1 e 2, considerando 3 Tipos de Processos Estocasticos (MGB, MRM e MRM com Tendência).

Tabela 21 - Sensibilidade da Razão entre Valor da Opção de Parada Temporária e o VPL Estático em Relação ao Percentual de OCD Evitado e a Volatilidade do Preço do Aço seguindo um MGB.

Tabela 22 - Sensibilidade da Razão entre Valor da Opção de Parada Temporária (Tipo 1) e o Valor do Projeto em Relação ao Drift de Tendência e a Volatilidade do Preço do Aço seguindo um MGB.

Tabela 23 - Diferença em Milhões de Reais entre o VPL Estático do MGB e do MRM, em Função de Níveis Distintos de Equilíbrio de Preços no MRM e Magnitude do Drift de Tendência no MGB.

Tabela 24 - Sensibilidade da Razão entre Valor da Opção de Parada Temporária (Tipo 1) e o Valor do Projeto em Relação aos Parâmetros de Velocidade da Reversão e a Volatilidade do Preço do Aço seguindo um MRM.

Tabela 25 - Sensibilidade da Razão entre Valor da Opção de Parada Temporária (Tipo 1) e o Valor do Projeto em Relação aos Parâmetros da Volatilidade e Nível de Equilíbrio do Preço do Aço seguindo um MRM.

Tabela 26 - Sensibilidade do Valor da Opção de Parada Temporária (Tipos I e II) em Milhões de Reais e do Percentual da Opção em Relação ao Valor do Projeto, considerando Níveis Distintos de Magnitude do Drift de Tendência dos Preços do Aço seguindo um MRM

Tabela 27 - Sensibilidade do Valor da Opção de Parada Temporária (Tipos I e II) e do Percentual da Opção em Relação ao Valor do Projeto, considerando Diferentes Valores Iniciais do Nível de Equilíbrio de Longo Prazo dos Preços do Aço seguindo um MRM com Tendência.

Tabela 28 - Sensibilidade da Razão entre Valor da Opção de Parada Temporária (Tipo 1) e o VPL Estático em Relação aos Parâmetros de Velocidade da Reversão e a Volatilidade do Preço do Aço seguindo um MRM com Tendência...

Tabela 29 - Opção de Troca de Tecnologia: Valor do Alto-Forno, VPL do AltoForno, VPL do Laminador, Valor Expandido do Laminador, Valor da Opção em Milhões de Reais.

Tabela 30 - Sensibilidade do Valor da Opção de Troca de Tecnologia considerando Diferentes Valores dos Drifts de Tendencia dos Processos de Preços do Aço seguindo o MGB 
Tabela 31 - Sensibilidade do Valor da Opção de Troca de Tecnologia considerando Diferentes Volatilidades dos Processos de Preços do Aço seguindo o MGB

Tabela 32 - Sensibilidade do Valor da Opção de Troca de Tecnologia considerando Diferentes Niveis de Correlacao entre os Processos de Preços do Aço seguindo um MGB.

Tabela 33 - Sensibilidade do Valor da Opção de Troca de Tecnologia considerando Diferentes Velocidads de Reversao dos Processos de Preços do Aço seguindo o MRM.

Tabela 34 - Sensibilidade do Valor da Opção de Troca de Tecnologia considerando Diferentes Niveis de Equilibrio de Longo Prazo dos Processos de Preços do Aço seguindo o MRM.

Tabela 35 - Sensibilidade do Valor da Opção de Troca de Tecnologia considerando Diferentes Niveis Inciais da Tendencia de Longo Prazo dos Processos de Preços do Aço seguindo o MRM com Tendencia... 